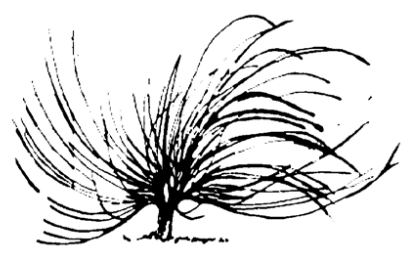

\title{
Giro en la formación docente en República Dominicana: ¿avance o retroceso en esta reforma? Un vistazo desde los derechos humanos
}

\author{
Basilio Florentino Morillo ${ }^{l}$ \\ Universidad Autónoma de Santo Domingo \\ República Dominicana \\ basilio_f@hotmail.com
}

\begin{abstract}
Resumen
En el ensayo, se analiza la actual reforma de la formación docente implementada en la República Dominicana. Se observa que esta prescinde de contenidos relacionados con la legislación y las normas de convivencia de la sociedad. Esta enmienda, impulsada por el Ministerio de Educación Superior, Ciencia y Tecnología, elimina la asignatura sobre legislación educativa, por lo que se aleja del mandato de la Declaración Universal de los Derechos Humanos, de la Constitución de la República, de la Ley de Educación Superior, Ciencia y Tecnología, de la Ley General de Educación y del mismo espíritu de la normativa que legaliza dicha reforma. Un cambio de esta naturaleza conlleva la generación de un déficit en la formación ciudadana, convivencia social y democracia. En consecuencia, la sociedad dominicana podría estar entrando en un probable alejamiento de la construcción de un Estado social de derechos, donde la ciudadanía conozca, defienda y practique sus privilegios, así como cumpla con sus deberes.
\end{abstract}

\section{(c) (1) $\Theta \Theta$}

Recibido: 4 de octubre de 2020. Aprobado: 1 de junio de 2021

http://dx.doi.org/10.15359/rep.16-2.6

1 Profesor Adjunto de la Universidad Autónoma de Santo Domingo. Doctor en Filosofía y Ciencias de la Educación por la Universidad de Barcelona, 2001. ORCID: https://orcid. org/0000-0001-7095-5564 
Palabras clave: Educación para los derechos humanos, estándares docentes, formación docente, legislación educativa, reforma de la educación

\begin{abstract}
The essay analyzes the current reform of teacher training implemented in the Dominican Republic. It dispenses with content related to society's legislation. This amendment promoted by the Ministry of Higher Education, Science, and Technology, which eliminates the subject on educational legislation, departs from the mandate of the Universal Declaration of Human Rights, the Constitution of the Republic, the Law of Higher Education, Science and Technology, the General Law of Education, and the same spirit of the regulations that legalize said reform. A change of this nature leads to the generation of a deficit in citizen training, social coexistence, and democracy. Consequently, the Dominican society could be entering a probable distance from the construction of a social state of rights, where citizens know, defend, and practice their privileges and fulfill their duties.
\end{abstract}

Keywords: education for human rights, educational legislation, educational reform, teacher training, teaching standards

\title{
Introducción
}

$\mathrm{E}$

1 presente trabajo tiene el propósito de analizar la actual reforma educativa impulsada por el Ministerio de Educación Superior, Ciencia y Tecnología (MESCYT), que introduce cambios en la formación docente en República Dominicana, mediante la Resolución 09-2015. Esta reforma parece alejarse de las recomendaciones de la Organización de las Naciones Unidas para que sus Estados miembros eduquen a su ciudadanía en el reconocimiento y respeto de los derechos humanos.

El enfoque de derechos constituye el elemento central de este estudio, para demostrar que la formación docente implementada actualmente en el país, si bien puede aportar avances en materia de profundización en los contenidos y la especialización, al prescindir de temas relacionados con el conocimiento de las normas educativas y 
sociales, puede estar generando un vacío instructivo esencial para la construcción ciudadana y el fortalecimiento de la convivencia social.

El país necesitaba una reforma de la formación docente como signo positivo para dar respuesta al déficit de calidad que ha caracterizado a la educación dominicana en los últimos 30 años, en busca de una mayor competitividad en la región, justo en materia educativa. Sin embargo, el debate universitario, en vez de centrarse en variables de calidad de la formación docente, parece haberse inclinado más hacia el reclamo de créditos y pulsos por el dominio de las especialidades. No obstante, las evidencias que se presentarán en este ensayo indican la necesidad de un cambio de horizonte formativo. Este encuentra su fundamento, por una parte, en los esfuerzos nacionales por incrementar la calidad de los aprendizajes de niños, niñas y adolescentes y, por la otra, en el deber irrenunciable del Estado dominicano de preparar a su ciudadanía en el marco universal de los derechos humanos.

\section{Algunos datos del contexto educativo y social dominicanos}

Las evaluaciones realizadas por el Ministerio de Educación (MINERD), que certifican los aprendizajes de la educación, mejor conocidas como pruebas nacionales, después de más de 20 años de impartirse de manera ininterrumpida, indican que el estudiantado que las toma no alcanza resultados satisfactorios. En promedio, no logran obtener una calificación superior al mínimo requerido para promover, que en primaria corresponde a $65 \%$ y en bachillerato al $70 \%$ de su valor. En la mayoría de los casos, los alumnos y las alumnas han quedado por debajo del 50 \% (El Día, 2014; Vargas, 2014).

A los bajos resultados que se indican, se les agrega la situación de estudiantes de bachillerato quienes no logran aprobar las pruebas nacionales, con el correspondiente impedimento de ingresar al nivel universitario; son colocados y colocadas en la "Sección 7", un concepto creado para esta población. En la actualidad, más de 69000 ciudadanos dominicanos y ciudadanas dominicanas (Educando, 2015) se encuentran varados y varadas en esa sección, sin poder continuar sus estudios en el nivel superior, ya que el propio sistema de evaluación implementado por el MINERD a principios de los años 90 no previó una posible salida a esta realidad. 
La Organización de las Naciones Unidas para la Educación, la Ciencia y la Cultura (UNESCO), a través del Laboratorio Latinoamericano de Evaluación de la Calidad Educativa (LLECE), en tres estudios realizados en los años 1997, 2006 y 2013, respectivamente (UNESCO, 1997, 2006 y 2013), ha demostrado que el alumnado dominicano de $3 .^{\mathrm{r}}$ y $6 .^{\circ}$ grado de la educación primaria corresponde al grupo de menor rendimiento en matemática, lengua española y ciencias en América Latina. De los países latinoamericanos participantes, la República Dominicana ha ocupado, específicamente, la última plaza.

En educación secundaria, la situación no es más alentadora. Así lo revela el Programa para la Evaluación Internacional de Alumnos (PISA) de la Organización para la Cooperación y el Desarrollo Económicos (OCDE), en el cual la República Dominicana viene participando desde el 2015. En sus dos últimos estudios, en los que participaron 72 y 79 países, respectivamente, el país obtuvo el último lugar en matemática y ciencias (OCDE, 2016; OECD, 2019). Este programa, que evalúa los conocimientos y habilidades necesarios para que estudiantes de 15 años puedan participar con éxito en la sociedad global, muestra deficiencias sustanciales en el estudiantado dominicano, lo que constituye una desventaja en un contexto global caracterizado por la competitividad.

En cuanto a la convivencia, en la que el conocimiento cívico es imprescindible para ejercer los roles de ciudadanía, también el gremio estudiantil de $8 .^{\circ}$ grado de la nación (hoy segundo de bachillerato) es el que refleja el menor conocimiento cívico del mundo (Schulz et al., 2010; Schulz et al., 2018), de acuerdo con sendas investigaciones realizadas por la Asociación Internacional para la Evaluación del Logro Educativo (IEA), en los años 2009 y 2016, respectivamente. De 38 países que participaron en el primer estudio y 24 en el segundo, el alumnado de República Dominicana ocupó el último lugar.

Estos resultados permiten deducir, como consecuencias lógicas, que si los saberes adquiridos por los y las estudiantes en áreas vitales para el desarrollo, el desenvolvimiento social y la convivencia son extremadamente bajos, como se ha evidenciado tanto en las evaluaciones internas como en las regionales y mundiales, entonces, los y las docentes que forman a esos niños, niñas y adolescentes en las aulas no universitarias tienen serias deficiencias en tales tipos de conocimientos. Esto, probablemente, constituye el principal argumento para la actual reforma educativa que ha impulsado el Ministerio de Educación 
Superior, Ciencia y Tecnología (MESCYT) de República Dominicana, junto al Consejo Nacional de Educación Superior, Ciencia y Tecnología (CONESCYT) y a las Instituciones de Educación Superior (IES). Esa enmienda se recoge en la Resolución No. 09-2015 del 9 de diciembre del 2015, que aprueba la normativa para regular la elaboración y el desarrollo de los programas de formación docente en República Dominicana, en el nivel preuniversitario. En ella, se otorga mayor peso a las disciplinas especializadas que a los componentes psicopedagógicos. Tanto es así que el título de egreso, en vez de ser en educación con mención en la especialidad de estudio, ahora será en la especialidad, con la mención en educación.

\section{La lupa sobre la reforma educativa precedente en la educación pre- universitaria y las correspondientes consecuencias sociales}

La reforma educativa precedente a la actual, impulsada por las autoridades dominicanas a principios de los años 90 , que se centró en la educación preuniversitaria, a través del primer Plan Decenal de Educación, tuvo un impacto positivo, en términos cuantitativos. Se incrementó la cobertura, la construcción de aulas y la dotación de materiales. También se introdujo un nuevo enfoque de enseñanza fundamentado en el constructivismo y la incorporación de ejes transversales como grandes temas que permeaban todo el currículo. Entre estos ejes se citan democracia y participación ciudadana; cultura dominicana; identidad y diversidad; educación para la salud; ciencia y tecnología; creatividad y desarrollo de los talentos, entre otros (SEEC, 1994).

El permear todo el currículo se entendió en esa reforma como temas que debían darse en todas las asignaturas, en todos los grados y niveles educativos. Es decir, que maestros y maestras debían ser expertos y expertas en metodologías transversales, para incorporar esas nuevas temáticas al contenido de las asignaturas habituales.

La reforma, basada en la estrategia de temas transversales, excluyó los contenidos filosóficos del nivel medio, incluso se eliminó el bachillerato en Filosofía y Letras, uno de los grandes desaciertos de esa enmienda. Además, retiró la educación moral y cívica del currículo, que luego incorporó a finales de la década, con la justificación de contrarrestar la pérdida de valores relacionados con los principios de la cultura local, para evitar, así, el deterioro de las buenas conductas en las familias dominicanas (Ordenanza 3, 1999). 
Pero, ¿los nuevos temas transversales incluyeron una metodología de trabajo, para que los y las docentes pudieran empoderarse de ella y ponerla en funcionamiento en el aula con sus estudiantes? ¿Se crearon contenidos integrados para que el maestro, en el momento de formar en ciencia, matemática, letras, sociales, idiomas y demás áreas, se instruyera, simultáneamente, en valores éticos, cívicos, democráticos, con miras a la convivencia, y sobre las normas compartidas que constituyen la base fundamental para la ese convivio humano entre la ciudadanía? Sin respuestas positivas a estas interrogantes, se estima que el fracaso se debió al funcionamiento de la estructura creada con el propósito de acompañar a los profesores y las profesoras en la implementación curricular. Romero (2016) expresa que las Comisiones de Construcción Curricular, mejor conocidas como las CCC, las cuales conformaban espacios de reflexión y análisis de las experiencias didácticas que demandaba la nueva reforma en la integración de los ejes, se convirtieron en contextos improductivos de reuniones y de pérdida de tiempo en el nivel nacional.

Entonces, ¿tendrá esa primera reforma global de la educación preuniversitaria alguna implicación para que niños y niñas de $8 .^{\circ}$ grado sean los y las de menor conocimiento cívico en el mundo? Del mismo modo, ¿se puede atribuir a esa reforma el hecho de que la República Dominicana ocupe el cuarto lugar del mundo y el primero de América Latina en muertes por accidentes de tránsito, según el informe de la Organización Mundial de la Salud (OMS) del 2018? ¿Tendrá que ver con el hecho de que las muertes de mujeres en manos de hombres vayan incrementándose año tras año y Dominicana llega a ser el quinto país de la región en este indicador, según el informe del 2019 de la Comisión Económica para América Latina y el Caribe (CEPAL)? ¿Ha incidido esa reforma en que el $88 \%$ de las mujeres que dieron a luz en la década completa del 2000 sean madres solteras (Pérez, 2015), o bien lo que equivale a decir que solo una de cada diez de las familias dominicanas está constituida formalmente?

¿Y qué comentar de los actos de delincuencia, violencia e inseguridad que vive la nación, así como del déficit democrático, el cual caracteriza a los partidos políticos, tanto los que gobernaron como los que gobiernan? ¿Tiene algo que ver el retiro del currículo de la educación preuniversitaria de las asignaturas relacionadas con la filosofía, en torno a los casos de corrupción evidenciados en el Estado dominicano, 
el desconocimiento de los derechos de salud de las personas envejecientes, las imposiciones de los y las congresistas que reprimen el derecho de las mujeres a decidir cuándo procrear y cuándo no? ¿Y qué decir de la justicia que descarga a presuntos políticos y presuntas políticas delincuentes o narcotraficantes políticos y políticas, cuando ella está para limitar los excesos del poder? ¿Tendrá que ver esa reforma con que el presupuesto nacional tome poco en cuenta las necesidades básicas humanas para su elaboración e implementación, o bien con el endeudamiento desenfrenado al que los sucesivos gobiernos están llevando la nación y que ya sobrepasa la astronómica cifra de 44000 millones de dólares, según la Dirección General de Crédito Público (2021), cuando en el 2000 era algo menos de 4200 millones, según la misma institución? ¿Tendrá esto enlace con la aprobación de una ley que penaliza el consumo de electricidad y, con ello, el desarrollo?

Aunque es imposible sostener que todos los problemas descritos sean causados exclusivamente por una reforma desacertada que buscó elevar la calidad de los aprendizajes en los años 90, parece haberse errado en la construcción de una ciudadanía más ética, más consciente tanto de sus derechos como de sus deberes $\mathrm{y}$, por tanto, una más democrática. Se remite a la idea de un conjunto ciudadano que reflexione y ponga como norte el bien común, así como el respeto y el reconocimiento de la dignidad humana. En este sentido, vale la pena comentar las acciones educativas puestas en marcha por la administración francesa, tras los atentados al semanario Charlie Hebdo, en enero del 2015, en el cual murieron 12 personas (El Mundo, 2015); los de París, en noviembre del mismo año, en el que fallecieron, al menos, 132 personas (BBC, 2015), y, más tarde, los de Niza, en julio del 2016, donde sucumbieron 84 personas (Yárnoz y Teruel, 2016). Todos esos ataques fueron atribuidos a la organización terrorista el "Estado Islámico".

Las autoridades francesas reforzaron de inmediato la enseñanza de los valores de la Revolución: libertad, igualdad y fraternidad (Vicente, 2015). De esa forma, han fortalecido la educación en principios éticos y laicos, que permiten el aprecio a la diversidad, el respeto a las diferencias y la tolerancia como aquellos que construyen convivencia armónica, lo cual no se realiza desde la escuela en el contexto dominicano, a pesar de los altos niveles de violencia que vive la sociedad actualmente. 


\section{La reforma de la formación docente a la luz de los derechos humanos}

Desde un enfoque de derechos, se recuerda que la Declaración Universal de los Derechos Humanos de 1948, pos Segunda Guerra Mundial - en la que se estima pudieron haber muerto hasta 70 millones de seres humanos (Halloran, 2015) - , expresa en su primer artículo, textualmente, que "Todos los seres humanos nacen libres e iguales en dignidad y derechos y, dotados como están de razón y conciencia, deben comportarse fraternalmente los unos con los otros". Ese artículo es precedido en el preámbulo por seis considerandos; los dos primeros constituyen la base de la necesaria civilización y la convivencia humana, cuando expresan que:

Considerando que la libertad, la justicia y la paz en el mundo tienen por base el reconocimiento de la dignidad intrínseca y de los derechos iguales e inalienables de todos los miembros de la familia humana;

Considerando que el desconocimiento y el menosprecio de los derechos humanos han originado actos de barbarie ultrajantes para la conciencia de la humanidad; y que se ha proclamado, como la aspiración más elevada del hombre, el advenimiento de un mundo en que los seres humanos, liberados del temor y de la miseria, disfruten de la libertad de palabra y de la libertad de creencias. (ONU, 1948, preámbulo, párr. 1 y 2)

Pero, también, dicha declaración —en el momento de ser proclamada por la Asamblea General de las Naciones Unidas- establece, previo al articulado que le da fisonomía, el compromiso de los Estados miembros de educar a la ciudadanía en estos derechos:

La Asamblea General proclama la presente Declaración Universal de Derechos Humanos como ideal común por el que todos los pueblos y naciones deben esforzarse, a fin de que tanto los individuos como las instituciones, inspirándose constantemente en ella, promuevan, mediante la enseñanza y la educación, el respeto a estos derechos y libertades, y aseguren, por medidas progresivas de carácter nacional e internacional, su reconocimiento y aplicación universales y efectivos, tanto entre los pueblos de 
los Estados Miembros como entre los de los territorios colocados bajo su jurisdicción. (ONU, 1948, preámbulo, párr. 6)

Eso significa que, para garantizar los derechos humanos de todas las personas, es necesario que estos se enseñen en los sistemas educativos de todos los Estados y naciones del mundo, a fin de que se pueda vivir en armonía, en paz, como gente civilizada, en oposición a la selva, donde el más fuerte o el que tiene más poder termina oprimiendo a todos los demás, o bien eliminando a otros con base en el uso de la fuerza. Por ello, las constituciones de todos los países miembros del Sistema de las Naciones Unidas están obligadas a incorporar el reconocimiento y el respeto a los derechos humanos, por mandato expreso de esta declaración.

En el caso de la nación dominicana, la constitución vigente (del 2015), en su artículo 26, reconoce que el país es un Estado miembro de la comunidad internacional (en referencia a la Organización de las Naciones Unidas) y como tal está abierto a la cooperación y a las normas del derecho internacional, entre las cuales se encuentran los derechos humanos. Por el reconocimiento de esta obligación, en su artículo 7, esa constitución afirma que:

la República Dominicana es un Estado Social y Democrático de Derecho, organizado en forma de República unitaria, fundado en el respeto de la dignidad humana, los derechos fundamentales, el trabajo, la soberanía popular y la separación e independencia de los poderes públicos. (Constitución de la República Dominicana, 2015, art. 7)

En dicho artículo, se asevera el compromiso del Estado con el respeto a la dignidad humana y a los derechos fundamentales de la ciudadanía reconocidos por la comunidad internacional. Sus líneas describen el apego de la nación a los derechos humanos, los cuales debe promover, garantizar y proteger para el libre ejercicio de los ciudadanos y las ciudadanas, en el marco del reconocimiento de los privilegios de las personas. De ese mismo modo, en su artículo 63, la Constitución define su papel frente al contenido educativo que se imparte en los establecimientos públicos y privados del país, cuando, además de reconocer la importancia del ejercicio de la carrera docente, en su inciso 13 establece que: 
Con la finalidad de formar ciudadanas y ciudadanos conscientes de sus derechos y deberes, en todas las instituciones de educación pública y privada, serán obligatorias la instrucción en la formación social y cívica, la enseñanza de la Constitución, de los derechos y garantías fundamentales, de los valores patrios y de los principios de convivencia pacífica. (Constitución de la República Dominicana, 2015, art. 63)

La cita anterior imprime un mandato constitucional al sistema educativo dominicano, que tiene la obligación de construir una ciudadanía informada de sus derechos y deberes como base que sustenta sus valores éticos y la convivencia social. En este sentido, la Ley General de Educación 66 del 1997, como forma de encarnar la aspiración constitucional, resalta, en su artículo 4, entre los principios y fines, el derecho a la educación, el respeto a la vida, la formación en valores, la cultura, la igualdad de oportunidades y otros no menos importantes que ellos. Esta ley establece los fines de la educación dominicana, en los que plantea la formación como el horizonte más elevado de las aspiraciones de la ciudadanía dominicana, en el marco de una nación civilizada, que no es otro que instruir para la libertad ciudadana, la justicia social y la dignidad humana. En los tres primeros incisos del artículo 5, esto queda definido cuando la ley sostiene que su finalidad es

formar personas, hombres y mujeres, libres, críticos y creativos, capaces de participar y constituir una sociedad libre, democrática y participativa, justa y solidaria; aptos para cuestionarla en forma permanente; que combinen el trabajo productivo, el servicio comunitario y la formación humanística, científica y tecnológica con el disfrute del acervo cultural de la humanidad, para contribuir al desarrollo nacional y a su propio desarrollo; formar ciudadanos amantes de su familia y de su Patria, conscientes de sus deberes, de sus derechos y de sus libertades, con un profundo sentido de responsabilidad y de respeto a la dignidad humana; educar para el conocimiento de la dignidad y la igualdad de derechos entre hombres y mujeres. (Ley General de Educación 66, 1997, art. 5) 
Es decir, se retoma el enfoque de derechos humanos como el único camino para asegurar una convivencia armónica entre los seres civilizados, donde, en el marco de la libertad y la justicia (esta última, como primera virtud social [Camps, 1990]), se permite la organización, en aras del conocimiento de los derechos personales y deberes frente a los y las demás, con los y las demás y en pro de los y las demás.

La orientación de derecho es nuevamente considerada en la misión de la Ley 139 del 2001, que creó el Sistema Nacional de Educación Superior, Ciencia y Tecnología. En el quinto inciso del artículo 11, esta plantea que una de las misiones de tal sistema consiste en

contribuir a proteger y consolidar los valores que conforman la identidad de la nación dominicana, velando por inculcar en los jóvenes los principios que sustentan una sociedad democrática, la defensa de la soberanía nacional, el respeto a los derechos humanos y la búsqueda de una sociedad más justa y equitativa. (Ley de Educación Superior, Ciencia y Tecnología 139, 2001, art. 11)

Entre los principales valores que sustentan una sociedad democrática, se encuentran la libertad y la justicia, que encarnan todas las leyes, como es el caso dominicano. Para que esa sociedad sea justa, entonces, las legislaciones que la conforman también deben serlo. Por eso, se acuña el respeto a los derechos humanos o "derechos de la gente", como recuerda Rawls (2001). Es decir, se apela al resguardo de la dignidad, la libertad en todas sus manifestaciones, la inclusión social, la justicia igualitaria y los otros derechos no menos importantes.

El enfoque de derechos humanos es refrendado en la Ley de Estrategia Nacional de Desarrollo 2030 y en el documento del Pacto Educativo del país. La primera lo pone de manifiesto cuando emula los privilegios de las personas consagrados en la Constitución y se fundamenta en una política transversal de derechos humanos. Esta ley expresa que

Todos los planes, programas, proyectos y políticas públicas deberán incorporar el enfoque de derechos humanos en sus respectivos ámbitos de actuación, a fin de identificar situaciones de vulneración de derechos, de discriminación o exclusión de grupos vulnerables de la población y adoptar acciones que 
contribuyan a la equidad y cohesión social. (Ley 1, que establece la Estrategia Nacional de Desarrollo de la República Dominicana 2030, 2012, art. 11)

El segundo, el Pacto Educativo como instrumento pautado por las Organizaciones de la Sociedad Civil bajo la coordinación del gobierno, confirma el enfoque abordado cuando plantea que la educación es el medio más pertinente para construir la cohesión social de la sociedad dominicana. Por ello, sobresale, de nuevo, el derecho a la educación y a la necesidad de construir una ciudadanía consciente de sus derechos y deberes, de conformidad con la Constitución, personas autónomas, solidarias, éticas, socialmente responsables y comprometidas con la igualdad de género, la atención a la diversidad, la protección y el uso sostenible de los recursos del medio ambiente (Palacio Nacional, 2014).

La misma normativa emitida por el CONESCYT para la reforma de la formación docente establece el compromiso del país en cuanto a formar ciudadanos y ciudadanas en el marco de valores éticos y el desarrollo de habilidades para la innovación, pero también para el respeto al medio ambiente, así como a su desarrollo holístico. Textualmente, indica que

nuestro principal compromiso como país es ayudar a formar estudiantes con grandes valores éticos; con capacidad de aprender permanentemente; con habilidades para cambiar, [...] respetuoso del medio ambiente; que desarrolle en los estudiantes un potencial intelectual, físico y espiritual para la innovación y la transformación; formado dentro de una estrategia de desarrollo holístico y enfatizando el desarrollo del conocimiento, con posesión de habilidades lingüísticas y tecnológicas que la sociedad futura demandará. (Resolución 9, 2015, pp. 3-4)

Siguiendo el mismo horizonte del párrafo anterior y apoyados en la Agenda 2030 de las Naciones Unidas para un desarrollo sostenible (PNUD, s. f.), la normativa enuncia en el perfil del nuevo docente la necesidad de garantizar en nuestros ciudadanos y nuestras ciudadanas una "educación inclusiva, equitativa y de calidad y promover oportunidades de aprendizaje durante toda la vida para todos" (Resolución 9, 2015, p. 4). Del mismo modo, traza la necesidad de contar con docentes bien 
cualificados y cualificadas pedagógicamente y en tecnologías, así como con la generación de entornos seguros, saludables e inclusivos. Asimismo, por su importancia para este trabajo, se reproduce aquí el contenido del párrafo de la normativa, en la cual se dice que el país

Requiere de alumnos educados por docentes capacitados y bien calificados, adecuadamente remunerados y motivados, de enfoques pedagógicos apropiados, apoyados por tecnologías de información y comunicación TICs apropiadas, así como también la creación de entornos seguros, saludables, receptivos al género, inclusivos y que cuenten con recursos adecuados, que faciliten el aprendizaje. (Resolución 9, 2015, p. 4)

Se enfatiza, en la cita anterior, la necesidad de que la ciudadanía dominicana sea formada de una manera inclusiva y equitativa, en entornos seguros y saludables. Esto significa que la educación que se reciba debe ser sin discriminación por condición de género, color, religión, preferencias sexuales, origen económico, geográfico o partidario; lo que esboza una educación para la tolerancia y la solidaridad (Florentino, 2016). Los entornos seguros y saludables hacen alusión al requerimiento de una convivencia efectiva, mediante la cual se respeten los derechos y se cumplan los deberes, libres de violencia y vicios, donde el norte sea la actuación ciudadana movida por el deber y la justicia, sin resentimientos, indignación ni remordimientos, en terminologías de Strawson (1995). Así es como se entiende, desde la óptica de derechos, lo de una educación inclusiva y equitativa en entornos seguros y saludables.

En definitiva, el Estado dominicano, ya sea por pertenecer al Sistema Internacional de las Naciones Unidas, por su Constitución o por sus leyes en el contexto educativo, está obligado a generar condiciones en el sistema educativo para la enseñanza de los derechos humanos, los valores éticos y la convivencia. Si eso es así, ¿cómo es que el Consejo Nacional de Educación Superior, Ciencia y Tecnología ha eliminado la asignatura Legislación Escolar del plan de formación de los y las docentes de todo el país? ¿Quién va a enseñar los derechos humanos y las leyes de convivencia a niños, niñas, adolescentes, adultos y adultas? ¿No se estarán sembrando las bases para una sociedad de actos de barbarie como a los que hace referencia el preámbulo de la Declaración 
Universal de los Derechos Humanos? ¿No se estará involucionando hacia formas más primitivas del reino animal? ¿Esto no es un absurdo?

Si en la formación de los y las docentes no se prevé una asignatura para trabajar la legislación y las normativas sociales más importantes para la ciudadanía y la democracia, en donde se trabajen la Constitución, que funda el Estado dominicano y establece los derechos constitucionales; la Ley General de Educación 66' 97, que norma el sistema educativo nacional; el Código para la Protección de los Derechos de la Niñez (Ley 136 del 2003), que protege a niños, niñas y adolescentes; el Reglamento del Estatuto del Docente (Decreto 639 del 2003), que establece la carrera docente, así como los derechos y los deberes de esta; las ordenanzas que tienen que ver tanto con la administración como con evaluación del currículo y la organización de la convivencia en los centros educativos, entonces, ¿cómo es que se obtendrá un Estado social democrático de derechos, cuando a quienes forman a la ciudadanía se les impide el derecho de conocer sus propios derechos?

En definitiva, la Resolución 09-2015, emitida por el MESCYT para la reforma de la formación docente en las IES, que excluye la asignatura de Legislación Escolar en todos los planes de formación docente, sin otra que la sustituya y asegure su espíritu, es inconstitucional. Por lo anterior, ella podría estar condenada al fracaso, ya que se distancia de la instrucción de una ciudadanía para seguir construyendo el Estado democrático, social y de derecho, establecido en la Constitución vigente de la República Dominicana; igualmente, aleja al sistema educativo de su compromiso de educar a la ciudadanía desde un enfoque de derechos humanos.

\section{Un acto de humildad: reconocer errores y superarlos}

Aún se está a tiempo de enmendar este error mayúsculo e incluso, en vez de retirar Legislación Escolar, se debería introducir más legislaciones, por ejemplo, las relacionadas con la educación vial, el libre acceso a la información, la seguridad social, la administración pública, entre otras. Es decir, la idea es buscar la formación de un ciudadano y una ciudadana consciente de sus derechos y sus deberes. Si la democracia no facilita la construcción de una ciudadanía respetuosa de las leyes, cuando son justas, ¿cómo se va a edificar ese Estado democrático, social y de derecho al que se alude en la Constitución? 
De seguir en esta dirección, en los próximos 10 años, los niveles de violencia actuales podrían ser recordados como juegos de niños frente a los que esperarían a la ciudadanía. Es necesario pensar en los niños y las niñas, la juventud, la vejez... en fin, en la gente, en la ciudadanía, en la humanidad, que son, en definitiva, los que construyen la sociedad. Sin el conocimiento y el respeto de las normas de convivencia, esa sociedad se vería forzada a ser violenta.

Es posible preguntarse: ¿qué se espera con esta reforma, en términos sociales, que deja fuera de sus planes formativos la legislación? Sin lugar a dudas, se estará educando una ciudadanía que, al no conocer las normas compartidas que han llevado a la sociedad a los niveles actuales de civilización, cada vez se alejará de esas reglas de convivencia, actuará por puro instinto animal, por puro utilitarismo egocéntrico, aumentando inmisericordemente la violencia que ya es en sí insoportable, el irrespeto a la dignidad humana y a los derechos de los y las demás. Así, estaríamos retornando a los tiempos de las cavernas, una vuelta a la selva, al salvajismo, al sálvese quien pueda. Sin embargo, es muy probable que este no sea el deseo de quienes dirigen la nación actualmente. Lo previo queda demostrado a través de la disposición presidencial que dispone, de forma obligatoria, la enseñanza de la Constitución en todos los centros educativos del país (Méndez, 2016). Esta es una razón más para enmendar el error que se ha cometido.

Es preciso afirmar con fuerza el fin de la humanidad, el de la civilización que se construye a través del sistema educativo, el cual no es otro que "construir más gente", aunque con ello, probablemente, se erija menos sabios, pues la sabiduría está en el reconocimiento y el respeto de los derechos de los y las demás y en vivir armoniosamente con ellos y ellas. Un científico es una persona que puede aportar mucho a la sociedad, pero sin valores éticos ni escrúpulos puede utilizar todos sus conocimientos para lesionar los derechos colectivos y saciar su ego, en perjuicio de la dignidad de innumerables grupos humanos.

Se debe ir en la dirección indicada por la Organización de las Naciones Unidas para la Educación, la Ciencia y la Cultura (UNESCO) en materia de educación, según expone Delors en su informe a este organismo, de 1996. Pues, para finalizar, de acuerdo con este autor, la educación a lo largo de la vida debe mover a la ciudadanía al aprendizaje para conocer, hacer, ser persona, pero también para vivir juntos y juntas. Vivir juntos y juntas solo se logra con base en el respecto 
y reconocimiento universal a las diferencias, como recuerda Taylor (1996) en su ética para la autenticidad, el respeto a los derechos de los y las demás y al cultivo de una actitud tanto de tolerancia como de aprecio a la diversidad. Eso se consigue al conocer, asumir y promover los derechos y deberes de las personas, pero no sacando la legislación de los planes de formación docente, que más de ser un error podría ser una condena para la sociedad dominicana.

\section{Conclusión}

El ensayo ha tratado de analizar la reforma de la formación docente en la República Dominicana, impulsada por el Ministerio de Educación, Ciencia y Tecnología, a finales del 2015. En sentido amplio, se pretendió determinar la enmienda representa un avance o un retroceso en la instrucción ciudadana. En esta línea, el análisis realizado mostró que, en vez de avanzar, el país se encamina hacia una visión educativa que toma distancia de un enfoque centrado en derechos, tal y como se recomienda desde la Organización de las Naciones Unidas. En la reforma, se retrocede en la construcción de una ciudadanía conocedora de sus derechos y deberes, ya que prescinde de espacios curriculares que permitan el conocimiento de los derechos humanos, la Constitución, la legislación educativa, al igual que las demás leyes aseguradoras de la cohesión y la convivencia social. La reforma, estrictamente, se aparta del mandato de las Naciones Unidas de enseñar los derechos humanos en los sistemas educativos de sus países miembros; unos derechos que enriquecen el acervo universal de la convivencia humana y la protección de privilegios inalienables que nos hacen ser y sentirnos personas.

\section{Referencias}

British Broadcasting Corporation (BBC). (2015, 16 de noviembre). Francia: lo que se sabe de los ataques reivindicados por Estado Islámico que dejaron al menos 132 muertos en París. BBC. https://www. bbc.com/mundo/noticias/2015/11/151113_paris_ataque_ep

Camps, V. (1990). Virtudes públicas. Espasa Calpe.

Comisión Económica de las Naciones Unidas para América Latina y el Caribe. (2019). Observatorio de igualdad de género. Indicadores destacados. Feminicidio. http://oig.cepal.org/es 
Constitución de la República Dominicana. (2015). Santo Domingo: Gaceta Oficial 10805. http:/extwprlegs1.fao.org/docs/pdf/ dom187716.pdf

Decreto 639, que establece el Estatuto del Docente. (2003). Santo Domingo: Presidencia de la República Dominicana. https://www. semma.gob.do/media/1741/decreto-no639-03-que-establece-el-reglamento-del-estatuto-docente.pdf

Delors, J. (1996). La educación encierra un tesoro. Informe a la UNESCO de la Comisión Internacional sobre la educación para el siglo XXI. Santillana-UNESCO.

Dirección General de Crédito Público. (2021). Deuda del sector público no financiero representa el $56.6 \%$ del PIB. https://www. creditopublico.gob.do/inicio/novedades? codigo $=20210205 \#: \sim:-$ text $=$ Deuda $\% 20$ de $1 \% 20$ sector $\% 20$ p $\%$ C $3 \%$ BAblico $\% 20$ no,Interno $\% 20$ Bruto $\% 20$ (PIB) $\% 20$ estimado 1

Educando. (2015). Tutorias para estudiantes pendientes de Pruebas Nacionales. ttp://otrasvoceseneducacion.org/archivos/25061

El Día. (2014, 11 de julio). Pruebas Nacionales con las mismas malas notas. Promedio de calificaciones en Matemáticas, Naturales y Sociales fue 50 de 100. El Día. http://eldia.com.do/ pruebas-nacionales-con-las-mismas-malas-notas/

El Mundo. (2015, 8 de enero). Al menos doce muertos en un tiroteo en la sede de Charlie Hebdo, el semanario francés que publicó las caricaturas de Mahoma. El Mundo. https:/www.elmundo.es/ internacional/2015/01/07/54ad132422601d72428b4577.html

Florentino, B. (2016). Educación ética para el profesorado: formación inicial y permanente. 3C Print.

Halloran, N. (2015). Los caídos de la Segunda Guerra Mundial. [Video]. https://www.youtube.com/watch?v=m5G01Y7XHPE\&feature=youtu.be

Ley de Educación Superior, Ciencia y tecnología 139. (2001). Santo Domingo: Gaceta Oficial 10097. https://siteal.iiep.unesco.org/ sites/default/files/sit_accion_files/do_3063_0.pdf

Ley General de Educación 66. (1997). Santo Domingo: Congreso Nacional. https://siteal.iiep.unesco.org/sites/default/files/sit_accion_files/siteal_republica_dominicana_0325.pdf

Ley 1, que establece la Estrategia Nacional de Desarrollo de la República Dominicana 2030. (2012). Santo Domingo: Gaceta Oficial 
10656. https://siteal.iiep.unesco.org/sites/default/files/sit_accion_files/siteal_republica_dominicana_0341.pdf

Ley 136, Código para el Sistema de Protección y los Derechos Fundamentales de Niños, Niñas y Adolescentes. (2003). Santo Domingo. https://pgr.gob.do/wp-content/uploads/2019/03/LeyNo.-136-03.pdf

Méndez, W. (2016, 6 de noviembre). Decreto dispone enseñar Constitución en escuelas. Listín Diario. https:// listindiario.com/la-republica/2016/11/06/441986/ decreto-dispone-ensenar-constitucion-en-escuelas

Ordenanza 3. Que modifica los Artículos 19, 23 y 24 de la Ordenanza 1'95 que establece el currículo para la Educación Inicial, Básica, Media, Especial y de Adultos del Sistema Educativo Dominicano. (1999). Consejo Nacional de Educación. http://idec.edu.do/ Archivos/Ordenanza\%2003-1999.pdf

Organization for Economic Cooperation and Development (OECD). (2019). PISA 2018. Results Combined executive Summaries. [Informe]. https://www.oecd.org/pisa/Combined_Executive_Summaries_PISA_2018.pdf

Organización de las Naciones Unidas (ONU). (1948). Declaración Universal de los Derechos Humanos. https://www.un.org/es/documents/udhr/UDHR_booklet_SP_web.pdf

Organización de las Naciones Unidas para la Educación, la Ciencia y la Cultura (UNESCO). (1998). Primer estudio internacional comparativo sobre Lenguaje, Matemática y factores asociados en tercero y cuarto grado. https://unesdoc.unesco.org/ark:/48223/ pf0000123130_spa

Organización de las Naciones Unidas para la Educación, la Ciencia y la Cultura (UNESCO). (2009). SERCE: Segundo estudio regional comparativo y explicativo: los aprendizajes de los estudiantes de América Latina y el Caribe. [Reporte técnico]. https://unesdoc. unesco.org/ark:/48223/pf0000190297

Organización de las Naciones Unidas para la Educación, la Ciencia y la Cultura (UNESCO). (2015). Informe de resultados TERCE: logros de aprendizaje. https://unesdoc.unesco.org/ark:/48223/ pf0000243532 
Organización Mundial de la Salud (OMS). (2018). Global status report on road safety 2018. [Informe]. https://www.who.int/ publications-detail/global-status-report-on-road-safety-2018

Organización para la Cooperación y el Desarrollo Económicos (OCDE). (2016). PISA 2015. Resultados clave. [Informe]. https://www. oecd.org/pisa/pisa-2015-results-in-focus-ESP.pdf

Palacio Nacional. (2014). Pacto Nacional para la Reforma Educativa en la República Dominicana 2014-2030. https://siteal.iiep.unesco.org/sites/default/files/sit_accion_files/siteal_republica_dominicana_0349.pdf

Pérez, O. (2015, 3 de septiembre). 88\% de niños nacidos desde 2001 son de madres solteras. El Caribe. https://www.elcaribe.com. do/2015/09/03/sur-donde-existen-mas-madres-solteras/

Programa de las Naciones Unidas para el Desarrollo (PNUD). (s. f.). Resolución aprobada por la Asamblea General el 25 de septiembre de 2015. Transformar nuestro mundo: Agenda 2030 para el desarrollo sostenible. [Documento]. https://www.undp. org/content/dam/argentina/Publications/Agenda2030/PNUDArgent-DossierODS.pdf

Rawls, J. (2001). El derecho de gentes y una revisión de la razón pública. Paidós.

Resolución 9-2015, Normativa para la Formación Docente de Calidad en la República Dominicana. (2015). Ministerio de Educación Superior, Ciencia y Tecnología (MESCYT). http://www.inafocam.edu.do/transparencia/index.php/base-legal/base-legal-normativas?download=710:normativa-para-laformacion-docente-de-calidad-en-la-rep-dom-dic-9-2015

Romero, P. (2016). La transformación curricular del 1995. Facetas Educativas, 1(1), 62-65.

Schulz, W., Ainley, J., Fraillon, J., Kerr, D. y Losito, B. (2010). Resultados iniciales del Estudio Internacional de Educación Civica y Ciudadana. https://www.iea.nl/sites/default/files/2019-04/ ICCS_2009_Initial_Findings_Spanish.pdf

Schulz, W., Ainley, J., Fraillon, J., Losito, B., Agrusti, G. y Friedman, T. (2018). Becoming Citizens in a Changing World. IEA International Civic and Citizenship Education Study 2016 International Report. https://link.springer.com/book/10.1007/978-3-319-73963-2 
Secretaría de Estado de Educación y Cultura (SEEC). (1994). Fundamentos del currículo Tomo II. SEEC.

Strawson, P. (1995). Libertad y resentimiento. Paidós.

Taylor, C. (1994). La ética de la autenticidad. Paidós.

UNESCO. (2013). Tercer estudio regional comparativo y explicativo (TERCE 2013). https://es.unesco.org/fieldoffice/santiago/llece/ TERCE2013

UNESCO. (2006). Segundo estudio regional comparativo y explicativo (SERCE 2006). https://es.unesco.org/fieldoffice/santiago/llece/ SERCE2006

UNESCO. (1997). Primer estudio regional comparativo y explicativo (PERCE 2006). https://es.unesco.org/fieldoffice/santiago/llece/ PERCE1997

Vargas, E. (2014, 21 de agosto). Resultados Pruebas Nacionales 2012-2014. Acento.com. https://acento.com.do/opinion/resultados-pruebas-nacionales-2012-2014-8166767.html

Vicente, A. (2015, 16 de enero). Cuando la escuela no es 'Charlie'. El Pais. http:/internacional.elpais.com/internacional/2015/01/16/ actualidad/1421438256 885270.html

Yárnoz, C. y Teruel, A. (2016, 18 de julio). El Gobierno francés eleva a 84 los muertos en el ataque con un camión en Niza. El Pais. $\quad$ https://elpais.com/internacional/2016/07/14/actualidad/1468532799_683242.html 